\title{
Can potassium citrate replace sodium bicarbonate and potassium chloride of oral rehydration solution?
}

\author{
M R ISLAM \\ International Centre for Diarrhoeal Disease Research, Bangladesh
}

SUMMARY Ninety four children aged less than 5 years with diarrhoeal dehydration and acidosis were treated randomly with either World Health Organisation (WHO) oral rehydration solution containing sodium chloride, potassium chloride, sodium bicarbonate and glucose or an oral solution with tripotassium citrate monohydrate replacing the sodium bicarbonate and potassium chloride in the WHO solution. Fifty five children (58\%) were hypokalaemic (potassium less than $3.5 \mathrm{mmol} / \mathrm{l}$ ) on admission. All but two in the citrate group were successfully treated. There were no significant differences in rehydration solution intake, stool output, gain in body weight, and fall in plasma specific gravity and haematocrit between the two treatment groups after 48 hours' treatment. Significant improvement in the serum potassium concentration was observed in the hypokalaemic children receiving potassium citrate solution compared with children receiving WHO solution after 24 and 48 hours' treatment. None developed hyperkalaemia. Although children receiving potassium citrate solution corrected their acidosis at a slower rate than the WHO solution group during the first 24 hours, by 48 hours satisfactory correction was observed in all. Tripotassium citrate can safely replace sodium bicarbonate and potassium chloride and may be the most useful and beneficial treatment for diarrhoea and associated hypokalaemia.

Although the principles of appropriate formulation of oral rehydration solution have been extensively studied, controversies still remain regarding the electrolyte composition. ${ }^{12}$ The current World Health Organisation (WHO) oral rehydration solution, which contains bicarbonate, has some practical disadvantages when packed and stored under climatic conditions of high humidity and temperature. In these circumstances bicarbonate reacts with glucose or sucrose resulting in a brownish discolouration which is less acceptable to patients. The potassium concentration $(20 \mathrm{mmol} / \mathrm{l})$ in WHO solution is not adequate for the treatment of undernourished children with depleted total body potassium who may lose more than $30 \mathrm{mmol}$ potassium/l of stool while suffering diarrhoea due to cholera, Escherichia coli, or rotavirus infection. ${ }^{3}$ Our previous study has shown that sodium citrate is as effective as sodium bicarbonate in correcting acidosis in diarrhoea. ${ }^{4}$

Tripotassium citrate monohydrate is a stable crystalline salt that will not cause discolouration so readily when mixed with glucose or sucrose and stored in packets under conditions of high humidity. Thus an oral rehydration solution containing $10 \mathrm{mmol} / \mathrm{l}(3.24 \mathrm{~g})$ of tripotassium citrate/l will provide both adequate alkali and a higher concentration of potassium than the WHO solution.

We have studied whether (a) potassium citrate in oral rehydration solution can replace sodium bicarbonate and potassium chloride adequately, (b) the increased concentration of potassium $(30 \mathrm{mmol} / \mathrm{l})$ corrects hypokalaemia better than WHO solution, and (c) the increased concentration of potassium causes hyperkalaemia in normokalaemic patients.

\section{Patients and methods}

This double blind, randomised study was carried out at the International Centre for Diarrhoeal Disease Research, Bangladesh from April to September 1982 on children below 5 years of age. These children were admitted with mild or moderate degrees of dehydration. ${ }^{5}$ Informed consent was obtained from parents or legal guardians of the children after the nature of the procedures had been fully explained.

The biochemistry laboratory personnel prepared the packets of oral rehydration solutions, confirmed their chemical concentrations, and coded them. Nursing staff dissolved the packets according to a random schedule before giving the solution to the patients. 
Children in the potassium citrate group received a solution containing sodium 90 , potassium 30 , chloride 90 , tribasic citrate 10 , and glucose $111 \mathrm{mmol} / \mathrm{l}$ (sodium chloride $3.5 \mathrm{~g}$, potassium citrate $3.24 \mathrm{~g}$, and glucose $20 \mathrm{~g} / \mathrm{l}$ ). Children in the WHO solution group received solution containing sodium 90 , potassium 20 , chloride 80 , bicarbonate 30 , and glucose 111 $\mathrm{mmol} / \mathrm{l}$ (sodium chloride $3.5 \mathrm{~g}$, potassium chloride $1.5 \mathrm{~g}$, sodium bicarbonate $2.5 \mathrm{~g}$, and glucose $20 \mathrm{~g} / \mathrm{l}$ ).

Patients were treated exclusively with an oral rehydration solution. Total volume given during the first six hours was $50 \mathrm{ml} / \mathrm{kg}$ body weight for mild and $100 \mathrm{ml} / \mathrm{kg}$ for moderate dehydration. ${ }^{5}$ Oral treatment was continued to match losses by stool and vomit losses until the diarrhoea stopped. Intake of solution and stool output were recorded at 8 hour intervals.

Patients who failed to be rehydrated or to maintain hydration on oral solution alone were considered as treatment failures and were excluded from the study. These patients were rehydrated with intravenous fluid. The criteria for failure of treatment were based on clinical assessment supported by intake and output balance and failure to gain body weight. Breast milk, when available, or diluted cows' milk (half strength) were allowed four hours after hospital admission.

Blood samples were drawn for estimation of haematocrit, plasma specific gravity (by Goldberg refractometer) and, electrolytes (by IL flame photometer) on admission and at 24 and 48 hours after the start of oral treatment. Adequate samples of stool and urine were sent simultaneously for estimation of the potassium concentration and serum electrolyte values. Stool samples were cultured on admission for Vibro cholerae, Salmonella $\mathrm{sp}$, Shigella $\mathrm{sp}$, and enterotoxigenic Escherichia coli. $E$ coli isolates were tested for both heat labile and heat stable enterotoxins. ${ }^{67}$ Rotavirus was tested by ELISA method. ${ }^{8}$

The nutritional state of the children was assessed by weight for age index-the discharge weight was determined as a percentage of the National Center for Health Statistics median for age and sex. ${ }^{9}$ Hypokalaemic children whose serum potassium concentration on admission was less than $3.5 \mathrm{mmol} / \mathrm{l}$ were considered as a separate group than the normokalaemic children for data analysis. Statistical tests were performed using the Student's $t$ test and $\chi^{2}$ test. Data from two children who failed treatment with citrate solution were excluded from analysis.

\section{Results}

Ninety four children aged under 5 years were studied. Fifty five of them were hypokalaemic (potassium less than $3.5 \mathrm{mmol} / \mathrm{l}$ ) and 39 were normokalaemic (potassium 3.5 to $5.5 \mathrm{mmol} / \mathrm{l}$ ), none were hyperkalaemic. Twenty seven hypokalaemic children received potassium citrate rehydration solution and 28 received WHO solution. Nineteen normokalaemic children received the potassium citrate and 20 the WHO solution. Most $(53 \%)$ of these children were between 7 and 12 months of age and showed signs of moderate malnutrition.

Clinical characteristics of the children at the time of hospital admission in relation to age, sex, duration of diarrhoea, body weight, nutritional status, and haematocrit were not different between the groups. About $50 \%$ of the children were admitted with signs of moderate dehydration and acidosis as determined by serum bicarbonate (less than $15 \mathrm{mmol} / \mathrm{l})$. All $48(100 \%)$ children receiving the WHO solution and 44 of $46(96 \%)$ children receiving potassium citrate solution were successfully rehydrated and maintained their hydration status well until diarrhoea stopped. The two in whom oral treatment failed (cholera patients) were withdrawn from the study. Although they drank sufficient rehydration solution they remained dehydrated and failed to gain body weight due to persistent vomiting and heavy purging (greater than $10 \mathrm{ml} / \mathrm{kg}$ per hour). They were subsequently treated with intravenous fluid. Frequency of vomiting in patients receiving

Table 1 Comparison of the intake of oral rehydration solutions (ORS) and stool output after 24 hours and 48 hours treatment with potassium citrate ORS and WHO ORS (values, mean (SD))

\begin{tabular}{|c|c|c|c|c|}
\hline & \multicolumn{2}{|c|}{ Hypokalaemic group $(K<3.5 \mathrm{mmol} / \mathrm{l})$} & \multicolumn{2}{|c|}{ Normokalaemic group (K $3.5-5.5 \mathrm{mmol} / \mathrm{l})$} \\
\hline & $\begin{array}{l}K \text { citrate } O R S \\
(n=27)\end{array}$ & $\begin{array}{l}\text { WHO ORS } \\
(n=28)\end{array}$ & $\begin{array}{l}K \text { citrate ORS } \\
(n=17)\end{array}$ & $\begin{array}{l}\text { WHO ORS } \\
(n=20)\end{array}$ \\
\hline \multicolumn{5}{|l|}{ ORS intake ( $\mathrm{ml} / \mathrm{kg})$} \\
\hline $\begin{array}{l}\text { First } 24 \text { hours } \\
\text { Second } 24 \text { hours }\end{array}$ & $\begin{array}{l}251 \cdot 3(130 \cdot 9) \\
195 \cdot 1(102 \cdot 6)\end{array}$ & $\begin{array}{l}300 \cdot 4(242 \cdot 8) \\
247 \cdot 6(275 \cdot 1)\end{array}$ & $\begin{array}{l}258 \cdot 5(101 \cdot 2) \\
186 \cdot 0(102 \cdot 5)\end{array}$ & $\begin{array}{l}266.7(104.4) \\
175.6(95 \cdot 3)\end{array}$ \\
\hline \multicolumn{5}{|l|}{ Stool output ( $\mathrm{ml} / \mathrm{kg})$} \\
\hline
\end{tabular}


Table 2 Comparison of body weight gain, plasma specific gravity, serum sodium bicarbonate and serum and urinary potassium on admission, and 24 and 48 hours after treatment with potassium citrate oral rehydration solution (ORS) or WHO ORS (values, mean (SD))

\begin{tabular}{|c|c|c|c|c|}
\hline & \multicolumn{2}{|c|}{ Hypokalaemic group $(K<3.5 \mathrm{mmol} / \mathrm{l})$} & \multicolumn{2}{|c|}{ Normokalaemic group $(K 3.5-5.5 \mathrm{mmol} / \mathrm{l})$} \\
\hline & $\begin{array}{l}\text { Potassium citrate } \text { ORS } \\
(n=27)\end{array}$ & $\begin{array}{l}\text { WHO ORS } \\
(n=28)\end{array}$ & $\begin{array}{l}\text { Potassium citrate } \text { ORS } \\
(n=17)\end{array}$ & $\begin{array}{l}\text { WHO ORS } \\
(n=20)\end{array}$ \\
\hline \multicolumn{5}{|c|}{ Body weight gain (\%) } \\
\hline At 24 hours & $5 \cdot 2(3 \cdot 3)$ & $5 \cdot 7(3 \cdot 8)$ & $4 \cdot 1(2 \cdot 7)$ & $2 \cdot 8(4 \cdot 1)$ \\
\hline At 48 hours & $5.7(3.9)$ & $6 \cdot 2(3 \cdot 7)$ & $3.6(2 \cdot 6)$ & $3.9(4.5)$ \\
\hline \multicolumn{5}{|c|}{ Plasma specific gravity } \\
\hline At 24 hours & $1.024(.002)$ & $1.024(.002)$ & $1.024(.001)$ & $1.025(.002)$ \\
\hline At 48 hours & $1.023(\cdot 002)$ & $1.023(\cdot 002)$ & $1.025(\cdot 001)$ & $1.025(\cdot 001)$ \\
\hline \multicolumn{5}{|c|}{ Serum sodium (mmol/l) } \\
\hline On admission & $12 \cdot 0(2 \cdot 7)$ & $13 \cdot 0(3 \cdot 9)$ & $13 \cdot 5(3 \cdot 6)$ & $13 \cdot 7(3 \cdot 8)$ \\
\hline At 24 hours & $15 \cdot 2(4 \cdot 5)$ & $18 \cdot 1(4 \cdot 8) \dagger$ & $14.9(5.2)$ & $17.9(3.2) \dagger$ \\
\hline At 48 hours & $18.4(3.8)$ & $19 \cdot 5(5 \cdot 7)$ & $18.6(5 \cdot 0)$ & $18.0(5.6)$ \\
\hline \multicolumn{5}{|c|}{ Serum potassium (mmol/l) } \\
\hline On admission & $3 \cdot 0(\cdot 4)$ & $2 \cdot 8(\cdot 5)$ & $4 \cdot 2(\cdot 6)$ & $4 \cdot 4(\cdot 6)$ \\
\hline At 48 hours & $4.6(1.0)^{*}$ & $3.9(.9)$ & $4.4(\cdot 8)$ & $4.5(\cdot 7)$ \\
\hline \multicolumn{5}{|c|}{ Urinary potassium (mmolll) } \\
\hline At 24 hours & $8.0(7 \cdot 5)^{\mathrm{a}}$ & $5 \cdot 3(4 \cdot 7)^{b}$ & $19 \cdot 1(11.9)^{\mathrm{c}}$ & $11 \cdot 2(8.9)^{\mathrm{d}}$ \\
\hline At 48 hours & $10 \cdot 5(8 \cdot 0)^{e}$ & $7 \cdot 3(4 \cdot 9)^{f}$ & $17.6(10 \cdot 7)^{\mathrm{g}}$ & $13 \cdot 1(11 \cdot 3)^{h}$ \\
\hline
\end{tabular}

*Hypokalaemia was corrected better at 24 hours $(\mathrm{P}<0 \cdot 02)$ and at 48 hours $(\mathrm{P}<0 \cdot 01)$ in children receiving potassium citrate ORS than in children receiving WHO ORS (Student's $t$ test).

†Acidosis was corrected better at 24 hours $(\mathrm{P}<0.02)$ in both hypokalaemic and normokalaemic children receiving WHO ORS than in children receiving potassium citrate ORS (Student's $t$ test).

a $v$ c $\mathrm{P}<0.05 ;$ b $v$ d $\mathrm{P}<0.01$; e $v$ g $\mathrm{P}<0.05$; f $v$ h $\mathrm{P}<0.05$.

potassium citrate solution was not significantly greater than in those receiving WHO solution. No significant inter-group difference in the intake of solution and output of stool was observed (Table 1).

A significant improvement in the serum potassium concentration was observed both at 24 hours $(\mathrm{P}<0.02)$ and at 48 hours $(\mathrm{P}<0.01)$ after the start of treatment in the group receiving potassium citrate rehydration solution.

Only three patients in the potassium citrate group compared with 11 in WHO group remained hypokalaemic 48 hours after the beginning of treatment. No patient developed hyperkalaemia during treatment. In both hypokalaemic and normokalaemic children receiving potassium citrate solution acidosis resolved more slowly than in those receiving WHO solution during the first 24 hours of treatment. By 48 hours, however, satisfactory correction of acidosis was observed in all children (Table 2). Serum sodium and chloride, stool potassium contents at 0 , 24 , and 48 hours showed no significant differences between groups. All patients passed urine satisfactorily within 12 hours. Hypokalaemic children conserved potassium significantly better than normokalaemic children (Table 2).

The aetiological agents identified in the patients were $V$ cholerae (2), Shigella sp (1), ETEC (8), and rotavirus (12) in the potassium citrate group, and $V$ cholerae (3), Shigella sp (1), ETEC (8), and rotavirus (12) in the WHO group.

\section{Discussion}

This study shows that potassium citrate salt can be used in place of sodium bicarbonate and potassium chloride in oral rehydration solution, thereby reducing the number of ingredients from four to three. This study further confirms the findings of Nalin and co-workers, that hypokalaemia can be treated satisfactorily by using a higher concentration of potassium without any danger of hyperkalaemia. ${ }^{10} \mathrm{Re}-$ latively fewer patients remained hypokalaemic after treatment with a higher concentration of potassium. This suggests that a higher concentration of potassium is necessary to treat hypokalaemia and associated diarrhoea, particularly in developing countries where malnutrition with associated total body potassium depletion is a major health problem. Hypokalaemic children excrete less potassium in urine as most of the potassium is conserved by the body to resolve the hypokalaemia.

The reason for delayed correction of biochemical acidosis in patients receiving potassium citrate solution is not fully understood and needs further investigations. Delayed resolution of acidosis may, however, have little practical importance. 
The cost effectiveness of the two types of solutions has been assessed: in Bangladesh 100 packets of potassium citrate solution will cost US $\$ 9.8$ and WHO solution US $\$ 8 \cdot 00$. This small increase in cost is negligible when we compare relative advantages of the two types of solution. Moreover, citrate based salt can be easily made in tablet form for convenient dispensing.

These advantages over the WHO oral rehydration solution have important implications in developing countries where diarrhoea, malnutrition, and hypokalaemia are interlinked and where there is great need for oral rehydration therapy.

This research was supported by the International Centre for Diarrhoeal Disease Research, Bangladesh. I thank Dr Thomas Butler for his suggestions and revisions of the paper, Mr Meer Md Ramzan Ali for secretarial work and the staff of the Clinical Research Unit for help and cooperation.

\section{References}

${ }^{1}$ Hirschhorn N. Single solution for oral therapy of diarrhoea. Lancet 1975;ii: 1049.

2 Griffith LSC, Fresh JW, Watten RH, Villaroman MP. Electrolyte replacement in paediatric cholera. Lancet 1967;i:1197-9.

3 Molla AM, Rahman M, Sarker SA, Sack DA, Molla A. Stool electrolyte content and purging rates in diarrhea caused by rotavirus, enterotoxigenic $E$. coli and $V$. cholerae in children. J Pediatr 1981;98:835-8.
${ }^{4}$ Islam MR, Samadi AR, Bardhan PK, Ahmed SM, Ali A. Oral rehydration therapy: efficacy of sodium citrate equals to sodium bicarbonate for correction of acidosis in diarrhoea. Gut 1984;25:900-4.

5 World Health Organization. Diarrhoeal diseases control programme. A manual for the treatment of acute diarrhoea. WHO/CDD/SER/80.2). Geneva: World Health Organization, 1981.

${ }^{6}$ Guerrant RL, Brunton LL, Schnaitman TC, Rebhun LI, Gilman AG. Cyclic adenosine monophosphate and alteration of Chinese hamster ovary cell morphology: a rapid sensitive in vitro test assay for the enterotoxins of Vibrio cholerae and Escherichia coli. Infect Immun 1974;10:320-7.

${ }^{7}$ Dean AG, Ching YC, Williams HR, Harden LB. Test for Escherichia coli enterotoxin using infant mice: application in a study of diarrhea in children in Honolulu. J Infect Dis 1972;125:407-11.

${ }^{8}$ Yolken RH, Kim HW, Clem T, et al. Enzyme-linked immunosorbent assay (ELISA) for detection of human reovirus-like agent of infantile gastroenteritis. Lancet 1977;ii:263-7.

9 National Center for Health Statistics. NCHS growth curves for children, birth-18 years, United States. Hyattsville, MD: National Center for Health Statistics, 1977: DHEW publication no (PHS) 78-1650 (Vital and health statistics, series 11, no 34).

10 Nalin DR, Harland E, Ramlal A, et al. Comparison of low and high sodium and potassium content in oral rehydration solutions. J Pediatr 1980;97:848-53.

Correspondence to Dr M R Islam, ICDDRB, GPO Box 128, Dhaka-2, Bangladesh.

Received 9 April 1985 